\title{
Transmission of Correlated Sources over Non-Orthogonal Gaussian MACs
}

\author{
Jiguang $\mathrm{He}^{\dagger}$, Iqbal Hussain ${ }^{\dagger}$, Markku Juntti ${ }^{\dagger}$, Tad Matsumoto ${ }^{\dagger \star}$ \\ ${ }^{\dagger}$ Centre for Wireless Communications, FI-90014, University of Oulu, Finland \\ ${ }^{\star}$ Japan Advanced Institute of Science and Technology (JAIST) 1-1 Asahidai, Nomi, Ishikawa, 923-1292, Japan \\ Email: \{jhe, iqbal.hussain, markku.juntti\}@ee.oulu.fi, matumoto@jaist.ac.jp
}

\begin{abstract}
We investigate the transmission of multiple correlated binary sources to a single destination over non-orthogonal Gaussian multiple access channels (MACs). By considering a binary codebook, we derive the admissible rate regions of the two-source Gaussian MAC. It is demonstrated that the admissible rate region increases as the correlation between the sources increases. Furthermore, we develop an iterative joint source channel decoding scheme based on systematic irregular lowdensity parity-check codes by exploiting the correlation between the two sources. The constituent decoders corresponding to each source are implemented in parallel via local iterations, exchanging extrinsic information with each other during the global iterations. Simulation results are provided to verify the performance improvement of the transmission of correlated sources compared to its independent sources counterpart.
\end{abstract}

Index Terms-Non-orthogonal Gaussian multiple access channel (MAC), joint source channel decoding (JSCD), systematic irregular low-density parity-check (LDPC) codes, log-likelihood ratio (LLR).

\section{INTRODUCTION}

Transmission of multiple correlated sources ${ }^{1}$ has a wide range of applications, for example, data collected from the geographically closely located sensor nodes in super dense sensor networks and videos captured in real-time monitoring systems. The reliable and/or efficient transmission of correlated sources is of great importance, especially for nonorthogonal multiple access channels (MACs). To the best of the authors' knowledge, only a limited number of literatures dealt with the issue of transmission over non-orthogonal MACs. For instance, Roumy and Declercq in [1] optimized the low-density parity-check (LDPC) codes for the transmission of two independent sources over non-orthogonal Gaussian MACs under the constraint of equal power allocation. This approach was extended to more generalized unequal power allocation scenarios in [2]. It is demonstrated that both the transmission schemes can achieve the Shannon limit within $0.1-0.6 \mathrm{~dB}$ without time sharing or rate splitting [3]. However, the transmission of correlated sources over MACs is yet to be investigated from both information-theoretic and practical code design perspectives.

This work has been performed in the framework of the FP7 project ICT-619555 RESCUE (Links on-the-fly Technology for Robust, Efficient and Smart Communication in Unpredictable Environments), which is partly funded by the European Union. This work is also partially supported by the network compression based wireless cooperative communication systems (NETCOBRA, No. 268209) project, funded by the Academy of Finland.

${ }^{1}$ The terms "source" and "user" are interchangeable throughout the paper.
From the information-theoretic perspective, the study of transmission of correlated sources was carried out within the scope of general sufficient and/or necessary conditions for the lossless or lossy transmission in [4]-[6]. Moreover, achievable rate regions of the correlated quadratic Gaussian two-encoder source coding problem were investigated in [7], [8]. Joint source channel coding (JSCC) schemes were explored for the transmission of multiple memoryless and inter-correlated Gaussian sources over Gaussian MACs in [9], [10]. Based on JSCC, the transmission of two binary correlated sources over independent additive white Gaussian noise (AWGN) channels was studied in [11]. Near Shannon/Slepian-Wolf limit performance was obtained when the perfect/estimated correlation information of the two sources was utilized at the receiver. However, the transmission of binary correlated sources over non-orthogonal MACs has not been fully addressed yet.

In this paper, we first derive the achievable rate regions for the two-user case under the assumption of a binary codebook, which can also be extended to the scenarios of larger number of users and/or larger codebooks. It is very important to analyze the achievable rate regions since super dense wireless networks require the achievable rate regions to eliminate the traffic flooding by properly allocating the rates to the users. Then, we investigate the consequences of the correlation between the sources over the derived achievable rate regions. The achievable rate regions expand as the correlation increases. In addition, we introduce a new iterative joint source channel decoding (JSCD) scheme based on the systematic irregular LDPC codes (without any source codes). It is tailored for the reliable transmission of correlated sources over non-orthogonal Gaussian MACs. The correlation information of the sources are exploited in the JSCD procedures to enhance the transmission performance. Theoretical analysis confirms significant performance gain of the proposed scheme compared to the one designed exclusively for the transmission of independent sources as verified by the numerical examples with a fixed rate pair.

The rest of the paper is organized as follows. The system model is given in detail in Section II. Under the constraint of a binary codebook, the achievable rate regions of transmission of independent and correlated sources are studied in Section III. Section IV provides a practical JSCD scheme, which takes the correlation between the sources into account. Simulation results are provided in Section V, and concluding remarks are drawn in Section VI. 


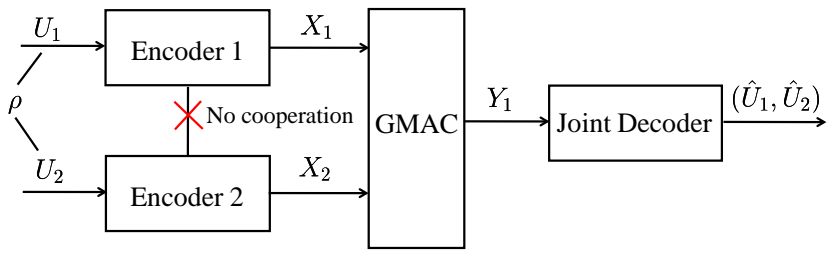

Fig. 1: A multiple access channel with correlated binary sources.

\section{System ModeL}

The transmission of two correlated, discrete, and memoryless sources over a Gaussian MAC is depicted in Fig. 1. The two sources are uniformly distributed with $\operatorname{Pr}\left(U_{i}=1\right)=$ $\operatorname{Pr}\left(U_{i}=0\right)=0.5$, for $i \in\{1,2\}$. The correlation between the two sources is represented by the bit flipping model, i.e., $U_{1}=U_{2} \oplus E$ with $\operatorname{Pr}(E=1)=p_{e}$ and $0 \leq p_{e} \leq 0.5$. Alternatively, the correlation coefficient can also be expressed as $\rho=1-2 p_{e}$. The two extreme cases are 1) fully correlated $(\rho=1)$ and 2$)$ independent $(\rho=0)$. Without being source coded, the two sources are directly and independently encoded to $X_{1}$ and $X_{2}$, and then synchronously transmitted over a Gaussian MAC with the received signal at the decoder as

$$
Y=\sqrt{P_{1}} X_{1}+\sqrt{P_{2}} X_{2}+Z,
$$

where $P_{i}$ is the transmission power of source $i$ and $E\left[\left|X_{i}\right|^{2}\right]=$ 1 , for $i \in\{1,2\}$. The additive noise $Z$ is Gaussian distributed with zero mean and unit variance (i.e., $\sigma^{2}=1$ ), expressed as $\mathcal{N}(0,1)$. Note that the correlation information is not exploited in the encoding process and no cooperation exists between the two sources. The discrete, memoryless, Gaussian MAC can be characterized by the transition probability $p\left(y \mid x_{1}, x_{2}\right)$, where $x_{1}, x_{2}$, and $y$ are the binary phase shift keying $\left(\right.$ BPSK $\left.^{2}\right)$ modulated version of the realizations of random variables (R.V.s) $X_{1}, X_{2}$, and $Y$, respectively. Depending on the applications, equal power $\left(P_{1}=P_{2}\right)$ or unequal power $\left(P_{1} \neq P_{2}\right)$ can be assigned to the two sources.

After receiving the transmitted data, an iterative JSCD scheme between the two constituent decoders by utilizing the correlation information is implemented to estimate the original message pair $\left(U_{1}, U_{2}\right)$ with the estimated version being $\left(\hat{U}_{1}, \hat{U}_{2}\right)$. The individual average bit error rate (BER) of each user is considered as a performance metric, which can be determined by

$$
P_{e}^{i}=\operatorname{Pr}\left\{U_{i} \neq \hat{U}_{i}\right\}, \text { for } i \in\{1,2\} .
$$

\section{Achievable Rate Regions}

For the transmission of independent sources, the MAC rate region can be expressed as [12]

$$
\begin{aligned}
R_{1} & \leq I\left(X_{1} ; Y \mid X_{2}\right), \\
R_{2} & \leq I\left(X_{2} ; Y \mid X_{1}\right), \\
R_{1}+R_{2} & \leq I\left(X_{1}, X_{2} ; Y\right),
\end{aligned}
$$

\footnotetext{
${ }^{2}$ The BPSK mapping $\{0 \rightarrow 1,1 \rightarrow-1\}$ is used throughout the manuscript.
}

where $R_{1}$ and $R_{2}$ denotes the encoding rates of user 1 and user 2, respectively. When the Gaussian codebooks are employed, the MAC region becomes

$$
\begin{array}{r}
R_{1} \leq \frac{1}{2} \log _{2}\left(1+P_{1}\right), \\
R_{2} \leq \frac{1}{2} \log _{2}\left(1+P_{2}\right), \\
R_{1}+R_{2} \leq \frac{1}{2} \log _{2}\left(1+P_{1}+P_{2}\right) .
\end{array}
$$

However, the Gaussian codebook is not applicable in practical communication systems. Therefore, we exploit a binary codebook, which can be achieved by virtue of BPSK, and derive its corresponding achievable rate regions. In the following subsections, we consider the transmission of both independent and correlated sources.

\section{A. Independent Sources}

We assume that the two binary sources are independent and uniformly distributed, that is, $\operatorname{Pr}\left(U_{i}=0\right)=\operatorname{Pr}\left(U_{i}=1\right)=0.5$, for $i \in\{1,2\}$. The joint probability mass function (PMF) can be expressed as $\operatorname{Pr}\left(U_{1}=i, U_{2}=j\right)=\frac{1}{4}$, for $i, j \in\{0,1\}$. We further assume that the encoding process does not introduce any additional correlation to the resulting encoded sequence $X_{i}$, for $i \in\{0,1\}$. Subsequently, the encoded sequence $X_{i}$ is independent and uniformly distributed, i.e., $\operatorname{Pr}\left(X_{i}=0\right)$ $=\operatorname{Pr}\left(X_{i}=1\right)=0.5$, for $i \in\{1,2\}$. The corresponding achievable rate region can be calculated as

$$
\begin{aligned}
R_{1} & \leq I\left(X_{1} ; Y \mid X_{2}\right)=H\left(X_{1}+Z\right)-H(Z) \\
& =-\int_{-\infty}^{\infty} p\left(y_{1}\right) \log _{2} p\left(y_{1}\right) d y_{1}-\frac{1}{2} \log _{2}(2 \pi e), \\
R_{2} \leq & I\left(X_{2} ; Y \mid X_{1}\right)=H\left(X_{2}+Z\right)-H(Z) \\
= & -\int_{-\infty}^{\infty} p\left(y_{2}\right) \log _{2} p\left(y_{2}\right) d y_{2}-\frac{1}{2} \log _{2}(2 \pi e),
\end{aligned}
$$

and

$$
\begin{aligned}
R_{1}+R_{2} & \leq I\left(X_{1}, X_{2} ; Y\right)=H\left(X_{1}+X_{2}+Z\right)-H(Z) \\
& =-\int_{-\infty}^{\infty} p\left(y_{3}\right) \log _{2} p\left(y_{3}\right) d y_{3}-\frac{1}{2} \log _{2}(2 \pi e),
\end{aligned}
$$

where $y_{1}=x_{1}+z, y_{2}=x_{2}+z$ and $y_{3}=x_{1}+x_{2}+z$ with $z$ being the realization of R.V. $Z$. The probability density functions (PDFs) of $p\left(y_{1}\right), p\left(y_{2}\right)$ and $p\left(y_{3}\right)$ in (9)-(11) are given by

$$
\begin{aligned}
p\left(y_{1}\right) & =\frac{1}{2 \sqrt{2 \pi}}\left(\exp \left(-\frac{\left(y_{1}-\sqrt{P_{1}}\right)^{2}}{2}\right)+\exp \left(-\frac{\left(y_{1}+\sqrt{P_{1}}\right)^{2}}{2}\right)\right) \\
p\left(y_{2}\right) & =\frac{1}{2 \sqrt{2 \pi}}\left(\exp \left(-\frac{\left(y_{2}-\sqrt{P_{2}}\right)^{2}}{2}\right)+\exp \left(-\frac{\left(y_{2}+\sqrt{P_{2}}\right)^{2}}{2}\right)\right) \\
p\left(y_{3}\right) & =\frac{1}{4 \sqrt{2 \pi}}\left(\exp \left(-\frac{\left(y_{3}-\sqrt{P_{1}}-\sqrt{P_{2}}\right)^{2}}{2}\right)\right. \\
& +\exp \left(-\frac{\left(y_{3}-\sqrt{P_{1}}+\sqrt{P_{2}}\right)^{2}}{2}\right)+\exp \left(-\frac{\left(y_{3}+\sqrt{P_{1}}-\sqrt{P_{2}}\right)^{2}}{2}\right) \\
& \left.+\exp \left(-\frac{\left(y_{3}-\sqrt{P_{1}}-\sqrt{P_{2}}\right)^{2}}{2}\right)\right)
\end{aligned}
$$




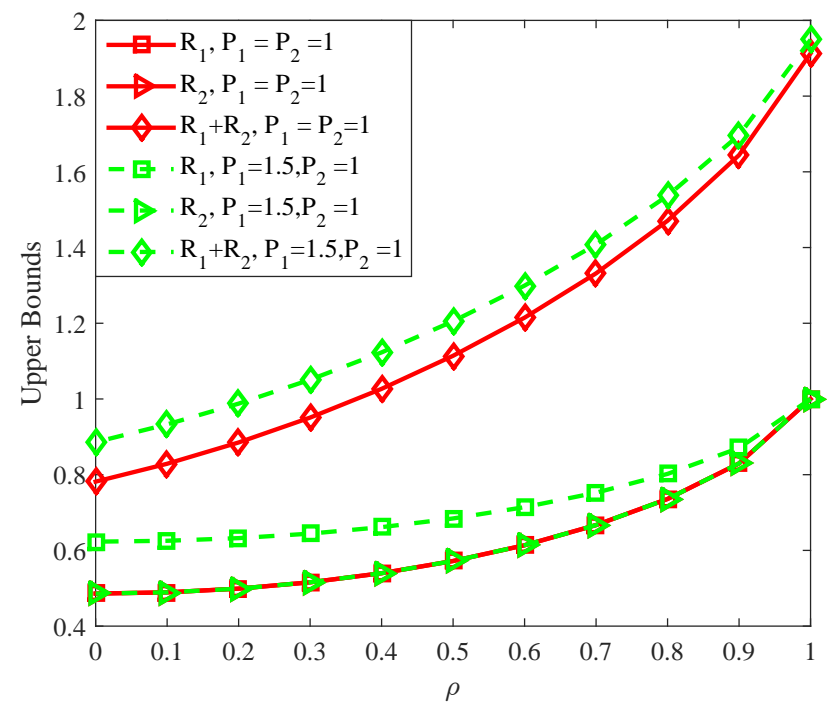

Fig. 2: The relationship between the upper bounds of the rates and correlation coefficient $\rho$.

\section{B. Correlated Sources}

In this subsection, we assume that the two sources are correlated before the encoding. We also assume the encoding process does not completely eliminate the correlation and can preserve a certain amount of the correlation information after encoding. This can readily be achieved via systematic channel codes. As a result, the codewords of the two sources are also correlated. Similar to the original sources, we model this correlation using bit flipping model, i.e., $X_{1}=X_{2} \oplus X$ with $\operatorname{Pr}(X=1)=p_{x}$. Since $X_{1} \leftrightarrow U_{1} \leftrightarrow U_{2} \leftrightarrow X_{2}$ forms a Markov chain, the amount of correlation between $X_{1}$ and $X_{2}$ can be upper bounded by

$$
I\left(X_{1} ; X_{2}\right) \leq I\left(U_{1} ; U_{2}\right),
$$

due to the data processing inequality. By solving (15), we can obtain the following relationship $0 \leq p_{e} \leq p_{x} \leq 0.5$. The achievable rate region of correlated sources can be derived as [12]

$$
\begin{aligned}
& R_{1} \leq I\left(X_{2}, Y ; X_{1}\right)=I\left(X_{1} ; Y \mid X_{2}\right)+I\left(X_{1} ; X_{2}\right), \\
& R_{2} \leq I\left(X_{1}, Y ; X_{2}\right)=I\left(X_{2} ; Y \mid X_{1}\right)+I\left(X_{1} ; X_{2}\right), \\
& R_{1}+R_{2} \leq I\left(X_{1}, X_{2} ; Y\right)+I\left(X_{1} ; X_{2}\right) .
\end{aligned}
$$

Since $X_{1}$ and $X_{2}$ can be regarded as the input and output of a binary symmetric channel (BSC) with crossover probability $p_{x}$, the common component (i.e., $\left.I\left(X_{1} ; X_{2}\right)\right)$ in (16)-(18) can be computed as $I\left(X_{1} ; X_{2}\right)=1-H_{b}\left(p_{x}\right)$, where $H_{b}\left(p_{x}\right)=$ $-p_{x} \log _{2}\left(p_{x}\right)-\left(1-p_{x}\right) \log _{2}\left(1-p_{x}\right)$. More details regarding the derivations of the upper bounds of $R_{1}, R_{2}$, and $R_{1}+R_{2}$ in (16)-(18) can be found in Appendix A. The three upper bounds are illustrated in Fig. 2 as a function of $\rho$.

\section{Examples}

The achievable rate regions for equal power case are shown in Fig. 3. Similarly, the achievable rate regions for unequal power case are depicted in Fig. 4. The achievable rate region

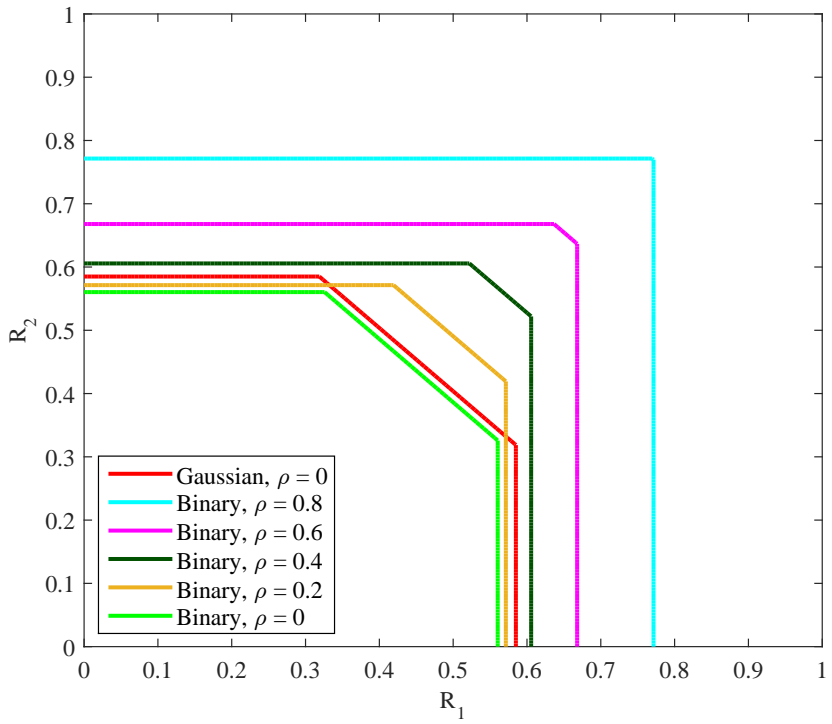

Fig. 3: Achievable rate regions of correlated sources when $P_{1}=$ $P_{2}=1.25$.

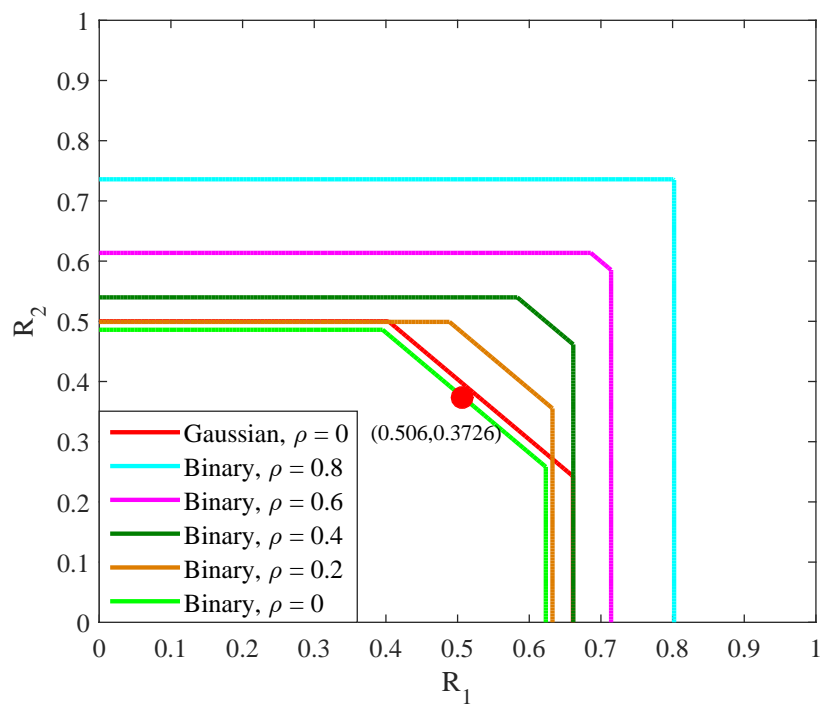

Fig. 4: Achievable rate regions of correlated sources when $P_{1}=1.5$ and $P_{2}=1$.

of Gaussian codebook is also provided as a benchmark. We observe from the figures that when the individual signal-tonoise ratios (SNRs), i.e., $P_{i} / \sigma^{2}$, for $i \in\{1,2\}$, are small, the achievable rate region of a binary codebook is close to its Gaussian counterpart for the transmission of independent sources. From the numerical results shown in Figs. 3 and 4, we can conclude that the achievable rate region becomes larger as the correlation coefficient increases (or $p_{e}$ decreases).

\section{Joint Source Channel Decoder}

We use two different and independent systematic irregular LDPC codes to encode the information bits of each user. Therefore, the codewords are in the form of $\mathbf{x}_{i}=\left[\mathbf{u}_{i} \mathbf{p}_{i}\right]$, for $i \in\{1,2\}$, where $\mathbf{u}_{i}=\left(u_{i}[1], \cdots, u_{i}\left[K_{i}\right]\right)$ with each entry generated by R.V. $U_{i}$ and $\mathbf{p}_{i}=\left(p_{i}\left[K_{i}+1\right], \cdots, p_{i}\left[N_{i}\right]\right)$ 


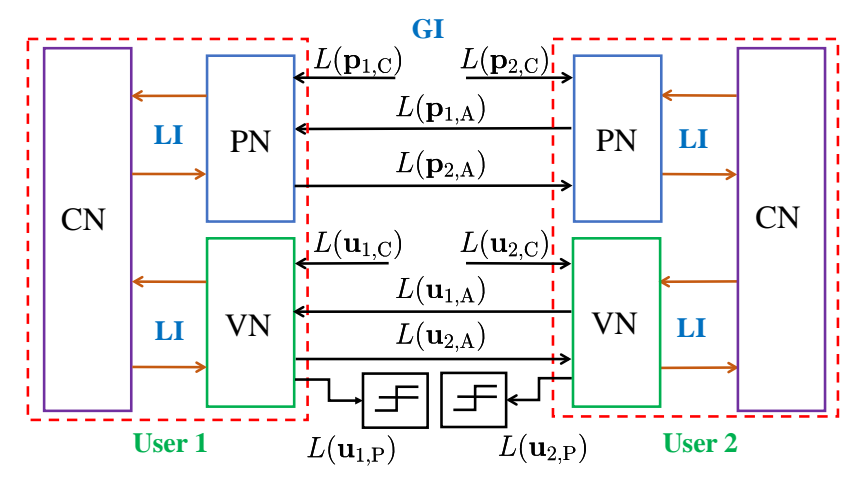

Fig. 5: Block diagram of the JSC decoder.

consists of the parity bits of the LDPC codes. The resultant individual coding rate can be computed by $R_{i}=K_{i} / N_{i}$. Then, BPSK modulated sequences are transmitted simultaneously by both users over a Gaussian MAC.

The block diagram of the joint source channel (JSC) decoder is shown in Fig. 5, where maximum a posteriori (MAP) algorithm [13] is applied to both decoders. Local iterations (LIs) within the constituent LDPC decoders and global iterations (GIs) between the constituent LDPC decoders are involved in the joint decoding procedures, exchanging the updated loglikelihood ratio (LLR) information. The notations utilized in Fig. 5 are defined as follows.

- VN, PN, CN are the variable, parity and check nodes of LDPC codes, respectively.

- $L\left(\mathbf{u}_{i, \mathrm{C}}\right)=\left[L\left(u_{i, \mathrm{C}}[1]\right), \cdots, L\left(u_{i, \mathrm{C}}\left[K_{i}\right]\right)\right]$ denotes the initial LLR from the channel for the information bits of user $i$, for $i \in\{1,2\}$.

- $L\left(\mathbf{p}_{i, \mathrm{C}}\right)=\left[L\left(p_{i, \mathrm{C}}[1]\right), \cdots, L\left(p_{i, \mathrm{C}}\left[N_{i}-K_{i}\right]\right)\right]$ denotes the initial LLR from the channel for the parity bits of user $i$, for $i \in\{1,2\}$.

- $L\left(\mathbf{u}_{1, \mathrm{~A}}\right)=\left[L\left(u_{1, \mathrm{~A}}[1]\right), \cdots, L\left(u_{1, \mathrm{~A}}\left[K_{i}\right]\right)\right]$ denotes the updated LLR for the information bits of user 1 from user 2 via GIs; it acts as a priori LLR of these information bits for the next round of LI. The same rule is applied to $L\left(\mathbf{u}_{2, \mathrm{~A}}\right)$.

- $L\left(\mathbf{p}_{1, \mathrm{~A}}\right)=\left[L\left(p_{1, \mathrm{~A}}[1]\right), \cdots, L\left(p_{1, \mathrm{~A}}\left[K_{i}\right]\right)\right]$ denotes the updated LLR for the parity bits of user 1 from user 2 via GIs; it acts as a priori LLR of these parity bits for the next round of LI. The same rule is applied to $L\left(\mathbf{p}_{2, \mathrm{~A}}\right)$.

- $L\left(\mathbf{u}_{i, \mathrm{P}}\right)=\left[L\left(u_{i, \mathrm{P}}[1]\right), \cdots, L\left(u_{i, \mathrm{P}}\left[K_{i}\right]\right)\right]$ denotes the $a$ posteriori LLR for the information bits of user $i$, for $i \in$ $\{1,2\}$.

The initial symbol-wise or bit-wise LLR information received from the channel can be calculated by (19) and (20) for user 1 , shown on the top of the next page. Similar calculations can also be applied to user 2 .

For better illustration, (19) and (20) can be further written by (21) and (22), from which we can observe that $L\left(u_{1, \mathrm{C}}[j]\right)$ is a function of $p_{e}{ }^{3}$ while $L\left(p_{1, \mathrm{C}}[j]\right)$ is independent of $p_{e}$. Each individual LDPC decoder proceeds in parallel via LIs based on the classical sum-product algorithm [14]. Moreover, the two decoders exchange extrinsic LLR information through

\footnotetext{
${ }^{3} \mathrm{We}$ assume that this type of correlation information can be perfectly available at the receiver side.
}

GIs, which is described in (23) and (24) using user 1 as an example. It is noted that both the extrinsic information from user 2 (denoted by $L\left(u_{2, \mathrm{E}}[j]\right)$ in (23) and (24)) and correlation information are considered for the updating of the information bits of user 1. However, only the extrinsic information from user 2 is involved for the updating of the parity bits of user 1 .

Finally, the hard decisions are made based on the a posteriori LLR information of $\mathbf{u}_{1}$ and $\mathbf{u}_{2}$.

$$
\hat{u}_{i}[j]=\left\{\begin{array}{l}
0, \text { if } L\left(u_{i, \mathrm{P}}[j]\right) \geq 0, \\
1, \text { if } L\left(u_{i, \mathrm{P}}[j]\right)<0 .
\end{array}\right.
$$

\section{Simulation Results}

The degree distribution of systematic irregular LDPC codes for the two users are [2]

$$
\begin{aligned}
\lambda_{1}(x) & =0.2429 x+0.3595 x^{2}+0.1433 x^{21}+0.0800 x^{22} \\
& +0.0631 x^{97}+0.1111 x^{98} \\
\lambda_{2}(x) & =0.1853 x+0.2762 x^{2}+0.0489 x^{11}+0.0705 x^{12} \\
& +0.0569 x^{31}+0.0567 x^{32}+0.3054 x^{199} \\
\rho_{1} & =\rho_{2}=x^{7} .
\end{aligned}
$$

The code rates of user 1 and user 2 are set to 0.506 and 0.3726 (marked with red dot in Fig. 4), respectively. The sum rate of the two users approaches the theoretical result, which is 0.8813 under the constraint of a binary codebook, derived in Section III. The gap between them is less than 0.03. The codeword length for each user is $10^{4}$. The number of LIs of each LDPC decoder is set to 20 followed by 1 GI in the iterative decoding process. The number of GIs in our simulations is 20 .

The BER performance of user 1 as a function of SNR is shown in Fig. 6. Likewise, the BER performance of user 2 as a function of its individual SNR is depicted in Fig. 7. As demonstrated in Section III, the achievable rate region expands with increase in the correlation between the sources. Consequently, the BER performance improves as the correlation increases for a fixed rate pair. The BER performance improvement is verified by the simulation results as demonstrated in Figs. 6 and 7. As shown in Fig. 6, there exists approximately $2.2 \mathrm{~dB}$ coding gain for user 1 at the BER level of $10^{-4}$ when $\rho$ changes from 0 to 0.8 . Similarly, at the same BER level, more than $2.5 \mathrm{~dB}$ coding gain can be achieved for user 2 when $\rho$ changes from 0 to 0.8 , as illustrated in Fig. 7.

\section{CONCLUSION}

We have derived the achievable rate regions for the transmission of correlated sources over Gaussian MACs under the constraint of a binary codebook. It has been demonstrated that the achievable rate region increases with the increase in the correlation between the sources. Moreover, we have introduced a practical iterative JSCD strategy by exploiting the correlation information of the two sources in the joint decoding process. Simulation results have verified the performance improvement of transmission of correlated sources compared to its independent sources counterpart. 


$$
\begin{aligned}
& L\left(u_{1, \mathrm{C}}[j]\right)=\ln \left(\frac{\operatorname{Pr}\left(u_{1}[j]=0, u_{2}[j]=0 \mid y[j]\right)+\operatorname{Pr}\left(u_{1}[j]=0, u_{2}[j]=1 \mid y[j]\right)}{\operatorname{Pr}\left(u_{1}[j]=1, u_{2}[j]=0 \mid y[j]\right)+\operatorname{Pr}\left(u_{1}[i]=1, u_{2}[j]=1 \mid y[i]\right)}\right), \quad \text { for } 1 \leq j \leq K_{1}, \\
& L\left(p_{1, \mathrm{C}}[j]\right)=\ln \left(\frac{\operatorname{Pr}\left(p_{1}[j]=0, p_{2}[j]=0 \mid y[j]\right)+\operatorname{Pr}\left(p_{1}[j]=0, p_{2}[j]=1 \mid y[j]\right)}{\operatorname{Pr}\left(p_{1}[j]=1, p_{2}[j]=0 \mid y[j]\right)+\operatorname{Pr}\left(p_{1}[i]=1, p_{2}[j]=1 \mid y[i]\right)}\right), \text { for } K_{1}+1 \leq j \leq N_{1} . \\
& L\left(u_{1, \mathrm{C}}[j]\right)=\ln \left(\frac{\exp \left(-\frac{\left(y[j]-\sqrt{P_{1}}-\sqrt{P_{2}}\right)^{2}}{2}\right)\left(1-p_{e}\right)+\exp \left(-\frac{\left(y[j]-\sqrt{P_{1}}+\sqrt{P_{2}}\right)^{2}}{2}\right) p_{e}}{\exp \left(-\frac{\left(y[j]+\sqrt{P_{1}}-\sqrt{P_{2}}\right)^{2}}{2}\right) p_{e}+\exp \left(-\frac{\left(y[j]+\sqrt{P_{1}}+\sqrt{P_{2}}\right)^{2}}{2}\right)\left(1-p_{e}\right)}\right), \text { for } 1 \leq j \leq K_{1}, \\
& L\left(p_{1, \mathrm{C}}[j]\right)=\ln \left(\frac{\exp \left(-\frac{\left(y[j]-\sqrt{P_{1}}-\sqrt{P_{2}}\right)^{2}}{2}\right)+\exp \left(-\frac{\left(y[j]-\sqrt{P_{1}}+\sqrt{P_{2}}\right)^{2}}{2}\right)}{\exp \left(-\frac{\left(y[j]+\sqrt{P_{1}}-\sqrt{P_{2}}\right)^{2}}{2}\right)+\exp \left(-\frac{\left(y[j]+\sqrt{P_{1}}+\sqrt{P_{2}}\right)^{2}}{2}\right)}\right), \text { for } K_{1}+1 \leq j \leq N_{1} .
\end{aligned}
$$

$L\left(u_{1, \mathrm{~A}}[j]\right)=\ln \left(\frac{\exp \left(-\frac{\left(y[j]-\sqrt{P_{1}}-\sqrt{P_{2}}\right)^{2}}{2}\right)\left(1-p_{e}\right) \exp \left(L\left(u_{2, \mathrm{E}}[j]\right)\right)+\exp \left(-\frac{\left(y[j]-\sqrt{P_{1}}+\sqrt{P_{2}}\right)^{2}}{2}\right) p_{e}}{\exp \left(-\frac{\left(y[j]+\sqrt{P_{1}}-\sqrt{P_{2}}\right)^{2}}{2}\right) p_{e} \exp \left(L\left(u_{2, \mathrm{E}}[j]\right)\right)+\exp \left(-\frac{\left(y[j]+\sqrt{P_{1}}+\sqrt{P_{2}}\right)^{2}}{2}\right)\left(1-p_{e}\right)}\right.$ for $1 \leq j \leq \min \left\{K_{1}, K_{2}\right\}$,

$L\left(p_{1, \mathrm{~A}}[j]\right)=\ln \left(\frac{\exp \left(-\frac{\left(y[j]-\sqrt{P_{1}}-\sqrt{P_{2}}\right)^{2}}{2}\right) \exp \left(L\left(p_{2, \mathrm{E}}[j]\right)\right)+\exp \left(-\frac{\left(y[j]-\sqrt{P_{1}}+\sqrt{P_{2}}\right)^{2}}{2}\right)}{\exp \left(-\frac{\left(y[j]+\sqrt{P_{1}}-\sqrt{P_{2}}\right)^{2}}{2}\right) \exp \left(L\left(p_{2, \mathrm{E}}[j]\right)\right)+\exp \left(-\frac{\left(y[j]+\sqrt{P_{1}}+\sqrt{P_{2}}\right)^{2}}{2}\right)}\right)$, for $\min \left\{K_{1}, K_{2}\right\}+1 \leq j \leq N_{1}$.

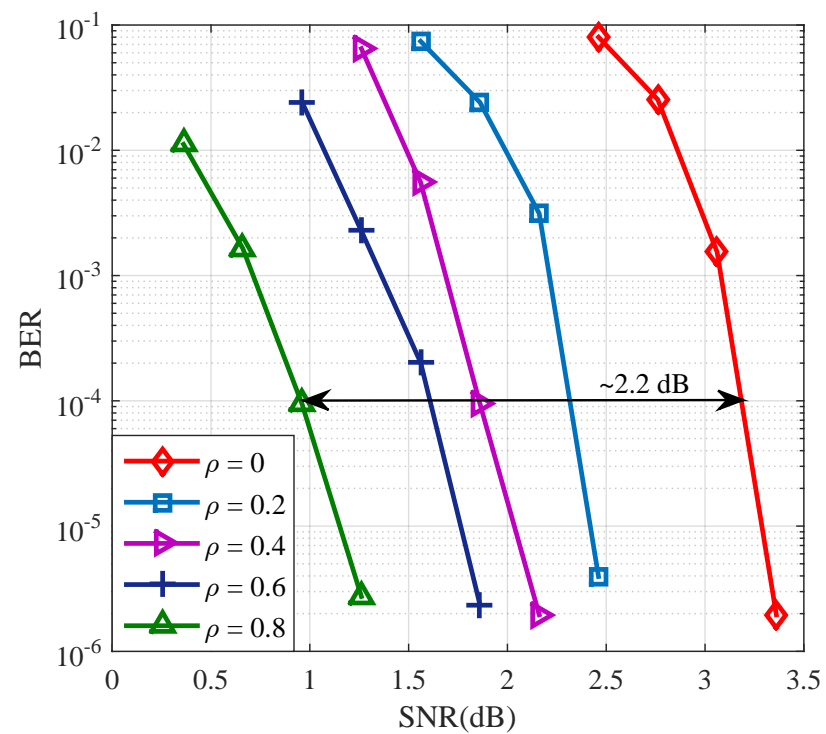

Fig. 6: BER performance of user 1 in terms of its individual SNR.

\section{APPENDIX A}

The conditional mutual information $I\left(X_{1} ; Y \mid X_{2}\right)$ in (16) can be further expressed as

$$
\begin{aligned}
I\left(X_{1} ; Y \mid X_{2}\right) & =H\left(Y \mid X_{2}\right)-H\left(Y \mid X_{1}, X_{2}\right) \\
& =H\left(X_{1}+Z \mid X_{2}\right)-H(Z) .
\end{aligned}
$$

The relationship between $X_{1}$ and $X_{2}$ can be considered as the input and output of a BSC with crossover probability $p_{x}$. We can easily get $\operatorname{Pr}\left(x_{1}=0 \mid x_{2}=0\right)=\operatorname{Pr}\left(x_{1}=1 \mid x_{2}=1\right)=$ $1-p_{x}$ and $\operatorname{Pr}\left(x_{1}=1 \mid x_{2}=0\right)=\operatorname{Pr}\left(x_{1}=0 \mid x_{2}=1\right)=p_{x}$. Considering the derivation in (29), the upper bound of $R_{1}$ can be calculated by

$R_{1} \leq H\left(X_{1}+Z \mid X_{2}\right)+I\left(X_{1} ; X_{2}\right)-H(Z)=\frac{1}{2} H\left(x_{1}+z \mid x_{2}=0\right)$

$+\frac{1}{2} H\left(x_{1}+z \mid x_{2}=1\right)+1-H_{b}\left(P_{x}\right)-\frac{1}{2} \log _{2}(2 \pi e)$

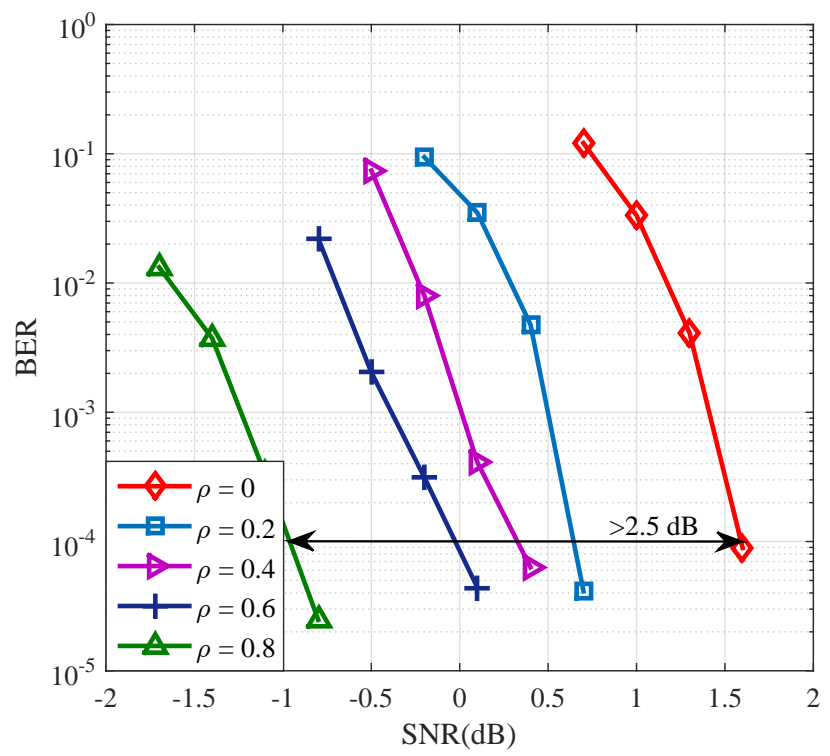

Fig. 7: BER performance of user 2 in terms of its individual SNR.

$=-\frac{1}{2} \int_{-\infty}^{\infty}\left(\frac{p_{x}}{\sqrt{2 \pi}} \exp \left(-\frac{\left(y+\sqrt{P_{1}}\right)^{2}}{2}\right)\right.$

$\left.+\frac{1-p_{x}}{\sqrt{2 \pi}} \exp \left(-\frac{\left(y-\sqrt{P_{1}}\right)^{2}}{2}\right)\right)$

$\log _{2}\left(\frac{p_{x}}{\sqrt{2 \pi}} \exp \left(-\frac{\left(y+\sqrt{P_{1}}\right)^{2}}{2}\right)+\frac{1-p_{x}}{\sqrt{2 \pi}} \exp \left(-\frac{\left(y-\sqrt{P_{1}}\right)^{2}}{2}\right)\right) d y$

$-\frac{1}{2} \int_{-\infty}^{\infty}\left(\frac{1-p_{x}}{\sqrt{2 \pi}} \exp \left(-\frac{\left(y+\sqrt{P_{1}}\right)^{2}}{2}\right)\right.$

$\left.+\frac{p_{x}}{\sqrt{2 \pi}} \exp \left(-\frac{\left(y-\sqrt{P_{1}}\right)^{2}}{2}\right)\right)$

$\log _{2}\left(\frac{1-p_{x}}{\sqrt{2 \pi}} \exp \left(-\frac{\left(y+\sqrt{P_{1}}\right)^{2}}{2}\right)+\frac{p_{x}}{\sqrt{2 \pi}} \exp \left(-\frac{\left(y-\sqrt{P_{1}}\right)^{2}}{2}\right)\right) d y$

$+1-H_{b}\left(P_{x}\right)-\frac{1}{2} \log _{2}(2 \pi e)$ 


$$
\begin{aligned}
& \leq-\frac{1}{2} \int_{-\infty}^{\infty}\left(\frac{p_{e}}{\sqrt{2 \pi}} \exp \left(-\frac{\left(y+\sqrt{P_{1}}\right)^{2}}{2}\right)\right. \\
& \left.+\frac{1-p_{e}}{\sqrt{2 \pi}} \exp \left(-\frac{\left(y-\sqrt{P_{1}}\right)^{2}}{2}\right)\right) \\
& \log _{2}\left(\frac{p_{e}}{\sqrt{2 \pi}} \exp \left(-\frac{\left(y+\sqrt{P_{1}}\right)^{2}}{2}\right)+\frac{1-p_{e}}{\sqrt{2 \pi}} \exp \left(-\frac{\left(y-\sqrt{P_{1}}\right)^{2}}{2}\right)\right) d y \\
& -\frac{1}{2} \int_{-\infty}^{\infty}\left(\frac{1-p_{e}}{\sqrt{2 \pi}} \exp \left(-\frac{\left(y+\sqrt{P_{1}}\right)^{2}}{2}\right)\right. \\
& \left.+\frac{p_{e}}{\sqrt{2 \pi}} \exp \left(-\frac{\left(y-\sqrt{P_{1}}\right)^{2}}{2}\right)\right) \\
& \log _{2}\left(\frac{1-p_{e}}{\sqrt{2 \pi}} \exp \left(-\frac{\left(y+\sqrt{P_{1}}\right)^{2}}{2}\right)+\frac{p_{e}}{\sqrt{2 \pi}} \exp \left(-\frac{\left(y-\sqrt{P_{1}}\right)^{2}}{2}\right)\right) d y \\
& +1-H_{b}\left(P_{e}\right)-\frac{1}{2} \log _{2}(2 \pi e) .
\end{aligned}
$$

Similar calculation can be applied to the upper bound of $R_{2}$, yielding

$$
\begin{aligned}
& R_{2} \leq-\frac{1}{2} \int_{-\infty}^{\infty}\left(\frac{p_{e}}{\sqrt{2 \pi}} \exp \left(-\frac{\left(y+\sqrt{P_{2}}\right)^{2}}{2}\right)\right. \\
& \left.+\frac{1-p_{e}}{\sqrt{2 \pi}} \exp \left(-\frac{\left(y-\sqrt{P_{2}}\right)^{2}}{2}\right)\right) \\
& \log _{2}\left(\frac{p_{e}}{\sqrt{2 \pi}} \exp \left(-\frac{\left(y+\sqrt{P_{2}}\right)^{2}}{2}\right)+\frac{1-p_{e}}{\sqrt{2 \pi}} \exp \left(-\frac{\left(y-\sqrt{P_{2}}\right)^{2}}{2}\right)\right) d y \\
& -\frac{1}{2} \int_{-\infty}^{\infty}\left(\frac{1-p_{e}}{\sqrt{2 \pi}} \exp \left(-\frac{\left(y+\sqrt{P_{2}}\right)^{2}}{2}\right)\right. \\
& \left.+\frac{p_{e}}{\sqrt{2 \pi}} \exp \left(-\frac{\left(y-\sqrt{P_{2}}\right)^{2}}{2}\right)\right) \\
& \log _{2}\left(\frac{1-p_{e}}{\sqrt{2 \pi}} \exp \left(-\frac{\left(y+\sqrt{P_{2}}\right)^{2}}{2}\right)+\frac{p_{e}}{\sqrt{2 \pi}} \exp \left(-\frac{\left(y-\sqrt{P_{2}}\right)^{2}}{2}\right)\right) d y \\
& +1-H_{b}\left(P_{e}\right)-\frac{1}{2} \log _{2}(2 \pi e) .
\end{aligned}
$$

We can calculate the upper bound of the sum-rate $\left(R_{1}+R_{2}\right)$ as

$$
\begin{aligned}
& R_{1}+R_{2} \leq I\left(X_{1}, X_{2} ; Y\right)+I\left(X_{1} ; X_{2}\right) \\
& =H(Y)-H\left(Y \mid X_{1}, X_{2}\right)+I\left(X_{1} ; X_{2}\right) \\
& =H(Y)-H(Z)+I\left(X_{1} ; X_{2}\right) .
\end{aligned}
$$

By definition,

$$
H(Y)=-E\left[\log _{2} p(y)\right]=-\int_{-\infty}^{\infty} p(y) \log _{2} p(y) d y
$$

where $p(y)$ is the PDF of $Y$, and it is in the form of

$$
\begin{aligned}
& p(y)=\frac{1-p_{x}}{\sqrt{2 \pi}} \exp \left(-\frac{\left(y+\sqrt{P_{1}}+\sqrt{P_{2}}\right)^{2}}{2}\right) \\
& +\frac{p_{x}}{\sqrt{2 \pi}} \exp \left(-\frac{\left(y+\sqrt{P_{1}}-\sqrt{P_{2}}\right)^{2}}{2}\right) \\
& +\frac{1-p_{x}}{\sqrt{2 \pi}} \exp \left(-\frac{\left(y-\sqrt{P_{1}}-\sqrt{P_{2}}\right)^{2}}{2}\right) \\
& +\frac{p_{x}}{\sqrt{2 \pi}} \exp \left(-\frac{\left(y-\sqrt{P_{1}}+\sqrt{P_{2}}\right)^{2}}{2}\right) .
\end{aligned}
$$

Let $p\left(y^{\prime}\right)$ be defined as

$$
\begin{aligned}
& p\left(y^{\prime}\right)=\frac{1-p_{e}}{\sqrt{2 \pi}} \exp \left(-\frac{\left(y^{\prime}+\sqrt{P_{1}}+\sqrt{P_{2}}\right)^{2}}{2}\right) \\
& +\frac{p_{e}}{\sqrt{2 \pi}} \exp \left(-\frac{\left(y^{\prime}+\sqrt{P_{1}}-\sqrt{P_{2}}\right)^{2}}{2}\right) \\
& +\frac{1-p_{e}}{\sqrt{2 \pi}} \exp \left(-\frac{\left(y^{\prime}-\sqrt{P_{1}}-\sqrt{P_{2}}\right)^{2}}{2}\right) \\
& +\frac{p_{e}}{\sqrt{2 \pi}} \exp \left(-\frac{\left(y^{\prime}-\sqrt{P_{1}}+\sqrt{P_{2}}\right)^{2}}{2}\right) .
\end{aligned}
$$

The sum-rate in (32) can be further expressed as

$$
\begin{aligned}
& R_{1}+R_{2} \leq H(Y)-H(Z)+I\left(X_{1} ; X_{2}\right) \\
& =-\int_{-\infty}^{\infty} p(y) \log _{2} p(y) d y+1-H_{b}\left(P_{x}\right)-\frac{1}{2} \log _{2}(2 \pi e) \\
& \leq-\int_{-\infty}^{\infty} p\left(y^{\prime}\right) \log _{2} p\left(y^{\prime}\right) d y^{\prime}+1-H_{b}\left(P_{e}\right)-\frac{1}{2} \log _{2}(2 \pi e) .
\end{aligned}
$$

It is not difficult to demonstrate that the upper bounds of $R_{1}, R_{2}$, and $R_{1}+R_{2}$ are decreasing functions of $p_{e}$. In other words, they are increasing functions of $\rho$, which is verified by the numerical results in Fig. 2.

\section{REFERENCES}

[1] A. Roumy and D. Declercq, "Characterization and optimization of LDPC codes for the 2-user Gaussian multiple access channel," EURASIP $J$. Wireless Comm. and Networking, vol. 2007, 2007.

[2] A. Balatsoukas-Stimming, "Design of LDPC codes for the two-user Gaussian multiple access channel," Master's thesis, Department of Electronic and Computer Engineering, Technical University of Crete, Chania, 2012.

[3] A. Amraoui, S. Dusad, and R. Urbanke, "Achieving general points in the 2-user Gaussian MAC without time-sharing or rate-splitting by means of iterative coding," in Proc. of IEEE ISIT, Jun. 2002, p. 334.

[4] T. Cover, A. Gamal, and M. Salehi, "Multiple access channels with arbitrarily correlated sources," IEEE Trans. on Inf. Theory, vol. 26, no. 6, pp. 648-657, Nov. 1980.

[5] R. Rajesh and V. Sharma, "Transmission of correlated sources over a fading multiple access channel," in 46th Annual Allerton Conference on Communication, Control, and Computing, Sep. 2008, pp. 858-864.

[6] V. Tan, "Transmission of correlated sources over a MAC: a Gaussian approximation-based analysis," in 50th Annual Allerton Conference on Communication, Control, and Computing (Allerton), Oct 2012, pp. 272277.

[7] A. Wagner, S. Tavildar, and P. Viswanath, "Rate region of the quadratic Gaussian two-encoder source-coding problem," IEEE Trans. on Inf. Theory, vol. 54, no. 5, pp. 1938-1961, May. 2008.

[8] A. Lapidoth and S. Tinguely, "Sending a bivariate Gaussian over a Gaussian MAC," IEEE Trans. on Inf. Theory, vol. 56, no. 6, pp. 2714 2752, Jun. 2010.

[9] R. Rajesh and V. Sharma, "A joint source-channel coding scheme for transmission of correlated discrete sources over a Gaussian multiple access channel," in Proc. of ISITA, Dec. 2008, pp. 1-6.

[10] P. Floor, A. Kim, T. Ramstad, I. Balasingham, N. Wernersson, and M. Skoglund, "On joint source-channel coding for a multivariate Gaussian on a Gaussian MAC," IEEE Trans. on Commun., vol. 63, no. 5, pp. 1824-1836, May. 2015.

[11] J. Garcia-Frias and Y. Zhao, "Near-Shannon/Slepian-Wolf performance for unknown correlated sources over AWGN channels," IEEE Trans. on Commun., vol. 53, no. 4, pp. 555-559, Apr. 2005.

[12] T. M. Cover and J. A. Thomas, Elements of Information Theory. New York, NY, USA: Wiley-Interscience, 1991.

[13] S. J. Johnson, Iterative Error Correction: Turbo, Low-Density ParityCheck and Repeat- Accumulate Codes. Cambridge University Press, Jan. 2010.

[14] F. Kschischang, B. Frey, and H.-A. Loeliger, "Factor graphs and the sum-product algorithm," IEEE Trans. on Inf. Theory, vol. 47, no. 2, pp. 498-519, Feb. 2001. 